\title{
GESTÃO INTEGRADA DE RESÍDUOS QUÍMICOS EM INSTITUIÇÕES DE ENSINO SUPERIOR
}

\author{
Patricia Carla Giloni-Lima* \\ Departamento de Biologia, Universidade Estadual do Centro Oeste, Rua Camargo Varela de Sá, 03, 85040-080 Guarapuava - PR, \\ Brasil \\ Vanderlei Aparecido de Lima \\ Engenharia Química, Universidade Federal de São Carlos, Rod. Washington Luiz km 235, 13565-905 São Carlos - SP, Brasil
}

Recebido em 9/5/07; aceito em 11/9/07; publicado na web em 7/8/08

\begin{abstract}
INTEGRATED MANAGEMENT FOR CHEMICAL RESIDUES IN ACADEMIC INSTITUTIONS. Since chemical residues management must be seriously considered at institutions of higher education and due to the relevance of this subject for students, this work proposes the involvement of the scientific community in the establishment of a program for management of chemical residues at universities, starting with a committee, to coordinate the process. The program should integrate the entire scientific community in an organized effort. The program involves legislative, educational and environmental management aspects, with environmental education as an important tool to integrate all the administrative areas of a chemistry department.
\end{abstract}

Keywords: tnstitutions of higher education; chemical waste management; chemical residues.

\section{INTRODUÇÃO}

Os Institutos e Departamentos de Química das Universidades, além de todas as unidades que utilizam produtos químicos em suas rotinas de trabalho, têm sido confrontados, ao longo de muitos anos, com o problema relacionado ao tratamento e à disposição final dos resíduos gerados em seus laboratórios de ensino e pesquisa. ${ }^{1}$ Isto ocorre devido a vários fatores: descentralização das instituições de ensino superior, atuação de mão-de-obra de elevada rotatividade, grande variação e mudanças nos processos e reagentes utilizados nas pesquisas, grande diversidade de resíduos e de volumes reduzidos, dentre outros. ${ }^{2}$ Outros elementos que contribuem para agravar o problema da gestão de resíduos químicos seriam: a ausência de um órgão gestor, a falta de consciência e visão de professores e pesquisadores, o descarte inadequado que reflete o descaso com o meio ambiente e a falta de estrutura e fiscalização de órgãos governamentais. ${ }^{3}$

No atual cenário, onde vários segmentos da sociedade vêm cada vez mais se preocupando com a questão ambiental, as universidades não podem mais sustentar esta medida cômoda de simplesmente ignorar sua posição de geradora de resíduos, ${ }^{4}$ não podendo mais ficar alheias às drásticas mudanças estruturais que vêm ocorrendo na legislação ambiental de nosso país. ${ }^{1}$

Devemos estar conscientes de que os benefícios oriundos de nossas atividades científicas e profissionais (publicações, patentes, reconhecimento científico, desenvolvimento de novos produtos e tecnologias) podem gerar, paralelamente, resíduos químicos de diversos graus de periculosidade, que podem necessitar de tratamento químico adequado, antes de serem enviados à disposição final. ${ }^{5}$

Outro importante aspecto a ser considerado, diz respeito às instituições de ensino superior como formadoras de mão-de-obra especializada. Precisamos despertar a atenção dos alunos de graduação e pós-graduação para essa questão e lhes fornecer as ferramentas básicas, que lhes permitam exercer suas atividades profissionais de forma limpa, além de estarmos contribuindo para a formação de cida-

*e-mail: pgiloni@gmail.com dãos compromissados com a nação. O primeiro passo para enfrentar esse desafio é assumir conscientemente a nossa responsabilidade para com os rejeitos gerados em nossos laboratórios e, depois, lutar para o estabelecimento de uma política institucional de gerenciamento desses resíduos. ${ }^{1}$

De acordo com os princípios apresentados, o objetivo deste artigo foi propor um fluxograma básico para implantação de um programa de gestão integrada de resíduos químicos em instituições de ensino superior, no qual se entende que somente com o envolvimento comprometido de toda a comunidade acadêmica (docentes, funcionários, estagiários e acadêmicos) é possível alcançar seus benefícios do ponto de vista ambiental, social, econômico, científico e educacional.

\section{FLUXOGRAMA BÁSICO}

O gerenciamento de resíduos químicos em laboratórios de ensino e pesquisa no Brasil começou a ser amplamente discutido nos anos 90, sendo de vital importância para as grandes instituições geradoras, incluindo as Universidades. ${ }^{3,5}$ Nos últimos anos, ações isoladas vêm sendo desenvolvidas por várias Instituições de Ensino Superior (notadamente públicas) e pela SBQ - Sociedade Brasileira de Química, visando aumentar a visibilidade dos problemas referentes ao gerenciamento de resíduos químicos. ${ }^{1,3}$ Mas apesar das diversas ações isoladas, essa questão precisa ser encarada coletivamente por toda a comunidade científica e pelos órgãos de fomento, dada sua relevância. ${ }^{1}$

A proposta do fluxograma básico (Esquema 1) a ser utilizado na estruturação e implementação de um plano de gestão integrada de resíduos químicos (PGIRQ) foi concebida levando em consideração alguns dos aspectos já discutidos anteriormente e considerando que a implementação ${ }^{4}$ de um programa de gestão de resíduos exige antes de tudo mudança de atitudes e, por isto, é uma atividade que traz resultados a médio e longo prazos, além de requerer alimentação contínua. Outro aspecto importante é o humano, pois o sucesso depende da participação e colaboração de todos os atores da unidade geradora, sendo de igual importância o apoio da instituição ao implementar e 


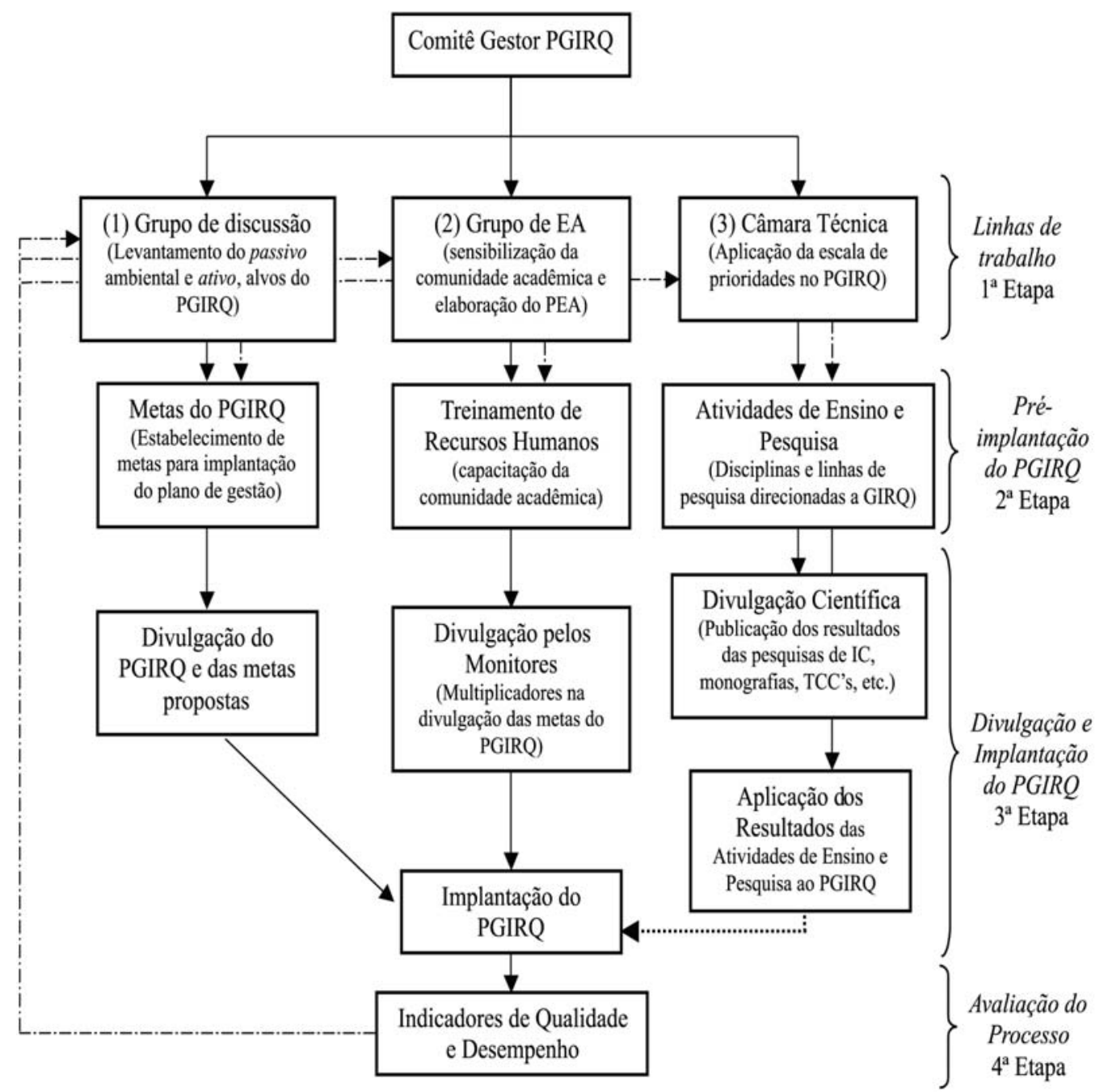

Esquema 1. Fluxograma básico para implantação do Plano de Gestão Integrado de Resíduos Químicos (PGIRQ) em Instituições de Ensino Superior. (EA = Educação Ambiental; PEA = Programa de Educação Ambiental; GIRQ = Gerenciamento Integrado de Resíduos Químicos; IC = Iniciação Científica; TCC's $=$ Trabalhos de Conclusão de Curso; linhas cheias = direção das linhas básicas de trabalho; linhas tracejadas = retroalimentação; linhas pontilhadas = aplicação direta dos resultados na implantação do PIGRQ)

sustentar o programa.

A utilização da hierarquia de atividades, ${ }^{4}$ ou escala de prioridades ${ }^{6}$ é outra prática importante, a qual pode ser utilizada independente das atividades da unidade geradora, permitindo prevenir a geração de resíduos sempre que possível, ou a minimização no volume de resíduos gerados e propiciando o reuso e a reciclagem, os quais reduzem o volume de resíduos a serem tratados e dispostos de maneira segura e adequada.

A estrutura geral do fluxograma consiste de quatro etapas que se iniciam a partir da constituição de um Comitê Gestor, o qual irá coordenar o desenvolvimento do processo para implantação do PGIRQ, desencadeado na formação das três Linhas Básicas de Trabalho $-1^{\text {a }}$ Etapa, a formação do (1) Grupo de Discussão, (2) Grupo de Educação Ambiental (EA) e (3) da Câmara Técnica. Os resultados dos trabalhos desenvolvidos na primeira etapa irão definir os principais pontos a serem trabalhados na $2^{\mathrm{a}}$ Etapa, a Pré-implantação do Plano de Gestão. Uma vez iniciada esta etapa, após ter reunido os requisitos básicos necessários, ou seja, definição de metas e objetivos do plano de gestão, bem como o treinamento mínimo para o cumpri-los, a $3^{\text {a }}$ Etapa é deflagrada - a Divulgação e Implantação do PGIRQ. As atividades de ensino e pesquisa por suas peculiaridades possuem um tempo próprio para serem concluídas e efetivar a aplicação de seus resultados (linha pontilhada do Esquema 1). A $4^{\mathrm{a}}$ Etapa, de Avaliação do Processo, representa o momento de reavaliação dos êxitos ou insucessos a partir dos Indicadores de Qualidade e Desempenho estabelecidos pelas linhas básicas de trabalho, para as quais poderá redirecionar seus resultados visando promover os ajustes necessários ao processo como um todo.

A partir da criação de um Comitê Gestor, é iniciado um processo de discussão para a formação de grupos de profissionais capacitados (membros do corpo docente e discente da instituição) para coordenar as três Linhas Básicas de Trabalho ( $1^{\text {a }}$ Etapa) na implantação do PGIRQ, com o objetivo de integrar a comunidade acadêmica e científica no processo, sendo:

(1) Grupos de discussão: poderão trabalhar no levantamento das informações contemplando dois tipos de resíduos: o passivo (o qual inclui desde restos reacionais, passando por resíduos sólidos e frascos sem rótulos) e o ativo (gerado continuamente, fruto das atividades rotineiras dentro da unidade geradora). A caracterização do passivo deve ser bem equacionada e utilizando-se de testes simples. ${ }^{4,7}$

(2) Grupo de Educação Ambiental: o objetivo de envolver os profissionais da área de Educação Ambiental (EA) é o de promover uma linha de trabalho que inicie um processo de sensibilização da 
comunidade acadêmica sobre a relevância da temática, antes mesmo do estabelecimento das metas do plano. Estes profissionais poderão trabalhar ainda na elaboração de um Programa de Educação Ambiental, a partir de informações fornecidas pelos grupos de discussão (1), com o intuito de auxiliar os processos de divulgação e treinamento da comunidade acadêmica.

(3) Câmara Técnica: este grupo poderá atuar no sentido de avaliar dentre as atividades práticas desenvolvidas nos laboratórios (em conjunto com os professores responsáveis pelas mesmas), a aplicação da escala de prioridades ${ }^{6}$ buscando as soluções mais viáveis dentro da sua realidade institucional. Este grupo pode atuar também como um gerador de temas para pesquisas futuras direcionadas a monografias, trabalhos de conclusão de curso e até na criação de linhas de pesquisa com o intuito de estruturar o gerenciamento de resíduos químicos perigosos ou não, na proposição de normas de segurança química, etc.; temas considerados relevantes e discutidos na "Carta de Niterói". ${ }^{1}$

A forma de constituição do Comitê Gestor e dos grupos que estejam dispostos a desenvolver as linhas de trabalho pode seguir uma dinâmica própria da instituição, ou ainda pode partir da criação de um fórum de discussão, o qual poderá representar um espaço aberto para discussões acerca do tema, para a circulação de informações a respeito da realidade institucional no tocante ao gerenciamento atual dos resíduos por ela gerados, para a troca de experiências e, principalmente, irão permitir avaliar o perfil dos profissionais que estariam mais aptos, sensibilizados e dispostos a se engajar na coordenação do processo (pertencendo ao Comitê Gestor) ou em cada uma das linhas básicas de trabalho (1, 2 ou 3). A participação de acadêmicos nesta fase seria de grande valia, pois teriam a oportunidade de demonstrar seu interesse em relação aos diferentes grupos de trabalho e atuar como multiplicadores, ou atuantes na pesquisa de informações e na geração de dados aplicados ao gerenciamento de resíduos químicos.

Na função de coordenar o processo de implantação do PGIRQ, o Comitê Gestor fica responsável por expor a proposta político-filosófica definida na sua constituição, seja no fórum de discussão ou em outra forma proposta pela instituição, promover reuniões periódicas entre os diferentes grupos de discussão que irão desenvolver as linhas básicas de trabalho, propiciando a integração de idéias e propostas a serem definidas por seus trabalhos e auxiliar na elaboração dos indicadores de qualidade e desempenho.

Uma vez estabelecidos os grupos responsáveis pela $1^{\mathrm{a}}$ etapa, o resultado dos trabalhos por eles desenvolvidos representa a $2^{\mathrm{a}}$ etapa do processo, a preparação para implantação do PGIRQ. Os grupos de discussão (1) devem promover o estabelecimento de metas e objetivos para implantação do plano de gestão.

Paralelamente, a promoção de um rol de atividades de EA pelo grupo (2) deve buscar a sensibilização da comunidade acadêmica, tornando-a mais receptiva ao processo de implantação do plano de gestão. O Programa de Educação Ambiental deve ser elaborado para atuar no sentido de que a implementação de um plano de gerenciamento traz resultados a médio e longo prazos, requerendo reeducação e persistência contínua. ${ }^{4,5}$ Nesse sentido, trata-se de conceber a EA como um instrumento no processo de gestão ambiental, onde suas atividades atuam visando a conscientização da comunidade para: a minimização da geração de resíduos, os processos de reciclagem, reuso e recuperação, e dentro desse sistema integrado de gestão de resíduos, representam importantes etapas na constituição de processos pautados em princípios ecológicos, de preservação ambiental e participação social.

O treinamento para capacitação da comunidade acadêmica poderá atuar na formação de recursos humanos especializados no gerenciamento e disposição final de resíduos químicos, tanto ao nível de graduação como de pós-graduação, o qual além de ampliar a qualificação técnica, poderá promover o fortalecimento da consciência ética e co-responsável tanto em termos de segurança química no trabalho como de responsabilidade ambiental. O público-alvo desta fase de treinamento pode envolver: técnicos dos laboratórios, funcionários da limpeza (em geral pertencentes a empresas terceirizadas e sem qualificação para atuarem na limpeza dos laboratórios, estando sujeitos a possíveis acidentes de trabalho), estagiários e acadêmicos de graduação e pós-graduação, conforme a necessidade local. A elaboração dos cursos poderia partir do próprio quadro funcional de professores da instituição, como de parcerias com outras instituições com experiência na área de treinamento de recursos humanos (SEBRAE, SENAI, etc.). Os cursos de treinamento poderiam envolver a prática de ações que visem a minimização do impacto ambiental e o risco às pessoas envolvidas com atuação direta ou indireta dos resíduos perigosos, dentre eles químicos, biológicos e radiativos, gerados nas atividades de ensino e pesquisa. ${ }^{1}$ A alocação de fundos poderia ocorrer por meio de projetos encaminhados aos órgãos de fomento que apóiam a pesquisa através de editais específicos, ou de parcerias com entidades públicas e privadas que podem ser beneficiadas futuramente com o oferecimento de cursos e programas de apoio à inserção social de pessoas sem qualificação, permitindo compartilhar com a sociedade conceitos fundamentais de gestão ambiental de resíduos, por meio de oferta de cursos de extensão à comunidade. ${ }^{8}$

Os problemas levantados pela Câmara Técnica na aplicação da escala de prioridades, ${ }^{6}$ dentro das atividades desenvolvidas na unidade geradora poderão nortear as atividades de ensino, pesquisa e extensão a serem desenvolvidas. A criação de disciplinas específicas ${ }^{9}$ ou relacionadas ao tema, ${ }^{10}$ e o oferecimento de estágios, ${ }^{8}$ são capazes de promover uma abordagem interdisciplinar, promovendo a formação do acadêmico numa perspectiva mais holística, trabalhando a capacitação técnica e a responsabilidade ambiental, além de promover atitudes éticas melhorando o perfil do futuro profissional. Com relação às atividades de pesquisa, inúmeras são as possibilidades de envolvimento de pesquisadores, técnicos e acadêmicos na geração de dados e informações que atendam aos preceitos básicos e aos aspectos de maior relevância com vistas a atender às exigências da legislação ambiental vigente, à minimização para redução do volume de resíduos com conseqüente redução de custos na destinação final adequada, além de buscar a segurança química no manuseio destes resíduos. Inúmeros também são os procedimentos já desenvolvidos na prevenção, minimização e reaproveitamento (reciclagem, recuperação e reutilização) dos resíduos químicos que podem estar sendo adaptados à realidade institucional e colocados em prática. ${ }^{4-12}$ Alguns procedimentos envolvem mudanças metodológicas, ${ }^{6}$ outros representam alternativas de baixo custo, tais como POA (Processos Oxidativos Avançados) para o tratamento de resíduos aquosos contaminados com compostos orgânicos potencialmente tóxicos, ${ }^{4}$ ou na degradação de corantes sintéticos. ${ }^{9}$ Alternativas baseadas em experiências adotadas em outros países ou desenvolvidas por pesquisadores brasileiros ${ }^{3-8,10}$ adaptadas à realidade de cada instituição, respeitadas às peculiaridades de cada resíduo, representam uma alternativa interessante.

A Divulgação ( $3^{a}$ Etapa) interna e externa do Plano de Gestão Integrada de Resíduos é fundamental para conscientização e difusão das idéias e atitudes que sustentarão o processo e deve-se procurar metas pouco ambiciosas e reais. ${ }^{4}$

Outro aspecto importante que deve ser considerado, uma vez que o processo está centrado na mudança de comportamento e atitudes, priorizando o lado humano do programa, ${ }^{4}$ diz respeito à Educação entendida como um dos instrumentos básicos e indispensáveis a sustentabilidade dos processos de gestão, no qual se deve considerar as questões de cidadania a partir do universo cognitivo, comunicativo e sociopolítico dos sujeitos que dão suporte às ações implementa- 
das, suas relações intersubjetivas, suas diferenças socioeconômicas, culturais e ideológicas. ${ }^{13}$

Nesse sentido é relevante destacar o fato de que há uma insustentabilidade da estrutura socioambiental das cidades, tanto das relações entre as pessoas, como das relações das pessoas com a natureza e com seus resíduos. Para que estas relações sejam viáveis, é necessário que haja Educação integrada no processo de Gestão Ambiental ${ }^{13}$ e que proporcione as condições necessárias para a produção e aquisição de conhecimentos e habilidades, e, que desenvolva atitudes, visando a participação individual e coletiva na gestão do uso de recursos ambientais e na concepção e aplicação das decisões que afetam a qualidade dos meios físico-natural e sociocultural. ${ }^{14}$

Podemos ainda utilizar os conceitos e pressupostos da Educação Ambiental, considerando o objetivo maior dessa área de conhecimento, que atua no sentido de repensar o estilo de vida humano, a partir da construção ampla de uma consciência crítica das relações sociedade/ natureza e indivíduo/sociedade e formar um cidadão atuante, dentro de uma proposta político-filosófica de mudança global do modelo societário e civilizacional vigente. ${ }^{15}$

A $4^{a}$ Etapa do processo é de extrema importância, pois irá promover uma Avaliação do Processo como um todo, por meio da utilização dos Indicadores de Qualidade e Desempenho, os quais poderão propiciar uma avaliação da eficiência do processo em função dos produtos que se pretende alcançar, permitindo o uso dos mecanismos de retroalimentação e recorrência para avaliar os sucessos e insucessos obtidos no processo, redirecionando-os de maneira a concretizar as metas e objetivos propostos.

Alguns elementos que podem auxiliar na estruturação dos indicadores de qualidade e desempenho são: ao identificar quais são os objetivos e metas a serem alcançados, definir quais formas de mensuração poderão estar sendo utilizadas e para cada indicador levantado, como estes serão calculados, com que freqüência ocorrerá a avaliação e a forma de interpretação dos resultados. Estes poderão fornecer subsídios para levantar os pontos específicos em que os objetivos e metas não estão sendo atingidos, permitindo avaliar em que linha (1,2 ou 3) os ajustes são necessários e retroalimentar as ações subseqüentes (linhas tracejadas no Esquema 1).

\section{CONCLUSÃO}

As discussões acerca do desenvolvimento sustentável e da busca crescente pela sustentabilidade ambiental fazem parte da nossa realidade social e institucional. Leonardo Boff fala com grande propriedade sobre este tema e cita que a categoria mestra deve ser a sustentabilidade e não o desenvolvimento, pois necessitamos da Terra, da sociedade e da vida humana sustentável, para então pensarmos em desenvolvimento.

Desta forma, as instituições de ensino necessitam criar condições de reflexão sobre mudanças que se fazem necessárias em relação: ao estilo de vida das pessoas, à redução do desperdício, à conscientização sobre a escassez dos recursos naturais e, principalmente, em relação à minimização da geração de resíduos e utilização dos 3 R's: redução, reutilização e reciclagem sempre que possível. As universidades enquanto formadoras de opiniões podem de forma direta ou indireta atuar nesse processo, através da formulação de políticas públicas em torno da valorização da participação dos cidadãos e reconhecimento das demandas e ações de resistência em face da degradação ambiental.

É baseada nessas premissas e no fato de que não havendo uma legislação especificamente definida para as peculiaridades dos resíduos gerados nas Instituições de Ensino Superior e nem uma fiscalização adequada para tal, que é apresentada esta proposta diferenciada à comunidade acadêmica convocando-a a refletir sobre o gerenciamento dos resíduos por ela gerados, motivados por várias razões: em busca de uma melhor da qualidade de vida, por uma exigência não só da legislação, mas também enquanto membros da sociedade; no fortalecimento da consciência ética e co-responsável em todos os níveis de hierarquia, onde prevaleça o interesse coletivo; na implantação de uma política ambiental e de um órgão gestor em nossa instituição e, na eliminação do descarte inadequado de resíduos e na redução do desperdício de materiais e recursos utilizados pela comunidade universitária.

As dificuldades no gerenciamento de resíduos gerados por instituições de ensino superior existem, bem como as dificuldades inerentes à implantação de um programa de gestão de resíduos, porém deve-se levar em consideração que os benefícios são de relevância nos níveis educacional, científico, social, ambiental e econômico.

\section{REFERÊNCIAS}

1. Gerbase, A. E.; Coelho, F. S.; Machado, P. F. L.; Ferreira, V. F.; Quim. Nova 2005, 28, 3 .

2. Izzo, R. M.; Chem. Health Saf. 2000, 5/6, 29.

3. Sassiotto, M. L. P.; Dissertação de Mestrado, Universidade Federal de São Carlos, Brasil, 2005.

4. Jardim, W. F.; Quim. Nova 1998, 21, 671.

5. Afonso, J. C.; Noronha, L. A.; Felipe, R. P.; Freidinger, N.; Quim. Nova 2003, 26, 602 .

6. Tavares, G. A.; Bendassolli, J. A.; Quim. Nova 2005, 28, 732.

7. Armour, M. A.; J. Chem. Educ. 1985, 65, 64.

8. Imbroisi, D.; Guaritá-Santos, A. J. M.; Barbosa, S. S.; Shintaku, S. da F.; Monteiro, H. J.; Ponce, G. A. E.; Furtado, J. G.; Tinoco, C. J.; Mello, D. C.; Machado, P. F. L.; Quim. Nova 2006, 29, 404.

9. Bendassolli, J. A.; Tavares, G. A.; Ignoto, R. de F.; Rosseti, A. L. R. M.; Quim. Nova 2003, 26, 578.

10. Alberguini, L. B. A.; Silva, L. C.; Rezende, M. O. O.; Quim. Nova 2003, 26, 291.

11. Tavares, G. A.; Bendassolli, J. A.; de Souza, G.; Nolasco, F. R.; Bonassi, J. A.; Batagello, H. H.; Quim. Nova 2004, 27, 320.

12. Schneider, J.; Wiskamp, V.; J. Chem. Educ. 1994, 71, 587.

13. Zaneti, I. C. B. B.; Sá, M. L. M. B.; Resumos do I Seminário da Associação Nacional de Pesquisa e Pós-Graduação de Sociedade e Ambiente, Campinas, Brasil, 2002.

14. Quintas, J. S.; Pensando e praticando a educação ambiental na gestão do meio ambiente, IBAMA: Brasília, 2000.

15. Loureiro, F.; Layrargues, P. P.; Castro, R. S.; Educação Ambiental: repensando o espaço da cidadania, $3^{\mathrm{a}}$ ed., Cortez: Rio de Janeiro, 2000 . 